\section{Selective attention and serial processing in briefly presented visual displays*}

\author{
CHARLES W. ERIKSEN and ROBERT L. COLEGATE \\ University of Ilinois, Urbana, Illinois 61803
}

Displays of eight capital letters were presented in a circular arrangement around a central fixation point at tachistoscopic durations. Under different experimental conditions, one or two indicators, presented either 250 msec before, simultaneously with, or after the display, designated the letters that $\mathbf{S}$ was to report. The arrangement of conditions permitted inferences as to the order in which $\mathbf{S}$ encoded the letters from the display. The results supported an interpretation in terms of a serial process by which the letters were encoded or transferred from iconic representation to a short-term memory.

If a human $S$ is presented eight letters simultaneously in a brief visual exposure, he is able subsequently to report only a few of the letters presented, typically four or less. However, if simultaneously with the display an arrow or similar indicator is used to designate one of the letters, the $S$ reports this letter with a high level of accuracy. To accomplish this, $S$ apparently brings into play some type of selective mechanism that enables him to effectively choose which of eight equally potent stimuli he will note or encode into his short-term store. This selective process or mechanism would seem to embody most, if not all, of the characteristics that are embodied in the concept of selective attention.

In prior work we have determined some of the characteristics of this selective process, as well as some of the variables of which it is a function. of major interest is the time required for the selection to occur. The pioneering works of Averbach and Coriell (1961) and Sperling (1960) have shown that a visual display may be available in the form of an icon for several hundred milliseconds beyond its exposure duration. To be effective, selectivity would seem to have to occur during the stimulus presentation or the duration of the icon, although slight performance gain might be attributable to the preferred order of recall from short-term memory.

Research indicates that the selective process requires between 200 and $300 \mathrm{msec}$ (Eriksen \& Collins, 1969; Averbach \& Coriell, 1961). In the Eriksen and Collins experiment, individual $\mathbf{S}$ performance was adjusted to approximately $75 \%$ correct with a multiitem display and a simultareous indicator. Then the experimental

*This investigation was supported by United States Public Health Service Research Grant MH-1206, United States Public Health Service Research Career Program Award K6-MH-22014, and Predoctoral Fellowship 1-FO1-MH-47201-01. manipulation consisted of presenting the indicator at various lead times before the occurrence of the display. Performance was found to increase until the indicator led the display by approximately $200 \mathrm{msec}$. This asymptotic value would indicate the maximum time required for precise selection to take place.

Averbach and Coriell (1961) obtained estimates of approximately $270 \mathrm{msec}$ for the selectivity process in an experiment using masking. Again a multiitem display with simultaneous indicator was presented, but a mask was applied to the indicated item at various times following termination of the display. The assumption was that if the item had already been processed into a short-term store, it could not be masked.

In other experiments the number and physical spacing of irrelevant or noise elements of the display were found to effect the efficiency of selective attention. Keeley (1967) had his Ss report a target Landolt C (gapped either at bottom or at top) embedded among noise Landolt Cs (gapped right or left). Even when an indicator wa. simultaneously presented, designating the target, $S$ 's performance was impaired when the number of noise elements in the display was greater than three or four. Similarly, Eriksen and Rohrbaugh (1970) found, in displays consisting of capital letters, that accuracy in reporting the designated or indicated letter decreased as the number of letters in the circular display increased from 4 to 12 . They further found that this effect of number was independent of the physical spacing between adjacent stimuli. When spacing between adjacent stimuli was held constant, performance still declined as a function of the number of irrelevant stimuli.

However, the Eriksen and Rohrbaugh results also showed that spacing in itself was a variable that effected the efficiency of the selective process. The closer the stimuli were spaced to each other, the poorer was performance in identifying the indicated target. Further investigation found this spacing effect to have at least two different components. If the stimuli were within a $1 / 4$ of a degree of angle of each other, interference was due apparently to impairment of acuity. Effects of adjacent contours on acuity at brief exposures had previously been found by Flom, Weymouth, and Kahneman (1962) when adjacent contours were $1 / 3$ of a degree of angle or less apart. However, Eriksen and Rohrbaugh found no contour interference (acuity) effects with stimuli spaced $.38 \mathrm{deg}$ of angle or further, a result that agrees with Collins (1970). But, since they also found spacing effects, independent of numerosity at distances of $.38 \mathrm{deg}$ and greater, it would appear that physical spacing of stimuli in a visual display has an effect on selectivity over and above that attributable to contour interference and acuity.

The present experiments were designed to provide further information on the process by which one or two of a set of equally potent stimuli is selected from a briefly presented visual display. A within-Ss design involving five experimental conditions was employed in Experiment 1. In all conditions, displays consisting of a circular arrangement of eight letters were briefly presented. There were two conditions that involved a single indicator and required a single response. In one, the $S$ was required to report the letter designated by a black line indicator that occurred simultaneously with the display. In the other single-response condition, he was instructed to report the letter diametrically opposite on the circular array to the letter designated by the simultaneous indicator.

The other three conditions required $S$ to report two of the display letters. In one of these conditions, two letters were designated by two separate indicators; in another condition, only a single indicator was employed, but it fell midway between two adjacent letters and $\mathbf{S}$ was instructed to report both of these. In the final condition, two indicators were again employed, but one indicator occurred $250 \mathrm{msec}$ before the onset of the letter display and the second occurred simultaneously with the display. Here again $\mathbf{S}$ was required to report both designated letters.

The rationale for the choice of these particular conditions was as follows: The single indicator with the single response may be viewed essentially as a base-level condition. By setting performance at $85 \%$ to $90 \%$ correct under this condition, through 
adjustment of exposure duration of the display, the effects of the other conditions can then be determined. The performance obtained with the two simultaneous indicators/two responses condition when compared with this base level provides information relative to the difficulty of locating two indicators in the display and noting or encoding two letters. If a performance decrement is found for this double-indicator condition, it could be attributable to the increased time required in locating the second indicator and encoding the response. However, any performance decrement also could reasonably be attributed to a serial encoding of the information. Locating the position designated by an indicator may consist of structuring in a spatial manner the display; once structured, no further time is required to go selectively to any position in the display. If this were the case, then any decrement in performance under this condition might be attributable to serial encoding of the letters. During the time required for encoding of the first letter, the icon of the display would have deteriorated sufficiently to lead to greater inaccuracies in the encoding of the second letter.

Information relative to these two possibilities can be obtained from the condition requiring two responses, but with a single indicator that falls midway between the two letters to be reported. Here $S$ has only one indicator to locate or apprehend. If performance under this condition is comparable to that obtained in the two simultaneous indicators/two responses condition, it would suggest that impairment relative to the base condition was due to the serial encoding of the display letters.

Further evidence on this point is provided in the condition where the first indicator occurs $250 \mathrm{msec}$ prior to the display. On the basis of prior research (Eriksen \& Collins, 1969), this should allow sufficient time for the location process for one of the letters to have occurred. Performance on the second letter in the display in this condition should then be a function only of the location time for that letter and its encoding.

The condition -where the $S$ reports only the letter diametrically opposite the position where the indicator occurs was included to provide some evidence on whether the internal scanning or encoding process has spatial limitations. Shaw (1969) has presented evidence suggesting that spaces or blank areas require a finite processing time.

\section{EXPERIMENT 1}

Subjects

Six female undergraduate students at the University of Illinois served as paid Ss. All had normal or corrected to normal vision.

\section{Apparatus and Stimuli}

Stimuli were displayed with a three-field Model G-A Scientific Prototype tachistoscope, which had been modified by replacing the manufacturer's bulbs with Sylvania F4T5/CWX fluorescent lamps. The luminance of each of the three fields was set at $1 \mathrm{~mL}$.

Thirty-two stimulus displays were constructed by using the capital letters $A, T, H$, and $Y$ twice each to fill eight positions on each display card. The arrangements of the letters in a circular array on the display cards were constrained in that the same letter could not appear in adjacent positions and that letter-position combinations occurred equally often. The letters were black and were obtained from Paratipe 1131618 Futura Bold. (Each letter subtended $.2 \mathrm{deg}$ of visual angle.) They were mounted on white vinyl cards. The letters were equally spaced, $.6 \mathrm{deg}$ of visual angle apart, around an imaginary circle $1.5 \mathrm{deg}$ of visual angle in diameter. All cards had the 12-, 3-, 6-, and 9-o'clock positions filled, plus the four intermediate positions.

Indicators consisted of black lines, $.5 \mathrm{deg}$ of angle long and $.1 \mathrm{deg}$ of angle wide, mounted on clear plastic cards. Each indicator was located with reference to the center of the display on an imaginary ray extending beyond the designated letter position, with the nearest end $.2 \mathrm{deg}$ of angle from the letter. One set of eight single indicators was constructed. A second set of 28 double indicators consisting of all possible combinations of positions taken two at a time was constructed. A third set of eight single indicators was constructed such that each indicator fell midway between adjacent positions at the same distance from the center of the display as the other indicators. The adaptation field contained a black fixation cross, subtending $.7 \mathrm{deg}$ of visual angle, mounted on the same white vinyl cards that were employed for the stimulus displays. The adaptation field was on at all times except when stimulation was provided in one of the other fields of the tachistoscope.

The procedure of mounting the indicators on clear plastic cards reduced the number of stimulus displays that had to be constructed. The clear plastic card with the indicator was inserted in the same fjeld as the stimulus display but in front of it. In the condition where one indicator preceded the display by $250 \mathrm{msec}$, the clear plastic indicator card was inserted in front of a blank vinyl stimulus card and presented in the other field of the tachistoscope. In order to maintain comparability of luminance and viewing conditions in all three tachistoscopic fields, a clear plastic sheet without indicator was also present in the indicator and adaptation field.

\section{Procedure}

Each $S$ was given two practice sessions. The first was devoted to identifying letters when a single indicator occurred simultaneously with presentation of the display. The second practice session was used to establish an exposure duration such that the $S$ could identify the indicated letter with approximately $90 \%$ accuracy under a single simultaneous indicator condition. In this second session, $S$ was also given 16 trials of practice on each of the five experimental conditions. Feedback was provided after each trial. Prior to initiating a trial, $S$ fixated on the cross in the adaptation field and when it was in clear focus pressed a switch to initiate the stimulus presentation.

Ten experimental sessions of five blocks of 16 trials each (one block in each condition per session) were run in counterbalanced order. Each session was preceded by $5 \mathrm{~min}$ of dark adaptation and warm-up employing a single simultaneous indicator. Feedback was given on each trial during warm-up, but once the experimental trials began feedback was provided only at the end of the trial block in the form of telling the $\mathbf{S}$ how many correct responses he had made. For each block of trials, each letter in each position occurred equally often.

The five experimental conditions were: single simultaneous indicator/single response, in which the indicator occurred with the display and $\mathbf{S}$ was required to report the letter occupying the indicated position; two indicators/two responses, in which two indicators occurred with the display and $S$ reported the letters occupying the designated positions with order of report in clockwise order; early and simultaneous indicators/two responses, in which one indicator appeared $250 \mathrm{msec}$ before the display for a duration equal to that of the display and a second indicator occurred with the display and $\mathbf{S}$ reported the letters in the order that they were indicated; single indicator/two responses, in which the indicator occurred with the display but was located midway between two letters and $S$ was required to report the letter on each side of the indicator in clockwise order; and opposite/single response, which was identical to the single simultaneous indicator/single response condition, except that the $S$ was instructed now to report only the 
Table 1

Mean Percent Correct Identifications of Indicated Letters for the Five Condit uns

\begin{tabular}{|c|c|c|c|c|c|c|c|}
\hline \multirow{2}{*}{$\begin{array}{l}\text { Single } \\
\text { Indicator, } \\
\text { ingle } \\
\mathbf{R} \text { sponse }\end{array}$} & \multicolumn{2}{|c|}{$\begin{array}{c}\text { Single } \\
\text { Indicator, } \\
\text { Two Responses }\end{array}$} & \multicolumn{2}{|c|}{$\begin{array}{c}\text { Two } \\
\text { Indicators, } \\
\text { Two Responses }\end{array}$} & \multicolumn{2}{|c|}{$\begin{array}{l}\text { Early and Simul- } \\
\text { taneous Indicators, } \\
\text { Two Responses }\end{array}$} & \multirow{2}{*}{$\begin{array}{l}\text { Opposite, } \\
\text { Single } \\
\text { Response }\end{array}$} \\
\hline & R1 & R2 & R1 & R2 & $\mathbf{R 1}$ & R2 & \\
\hline 87 & 81 & 83 & 81 & 76 & 88 & 72 & 79 \\
\hline
\end{tabular}

le:ter diametrically opposite the letter indicated.

On the basis of the second practice session, an exposure duration was determined for each $S$ that yielded ar:proximately $90 \%$ report accuracy for the single simultaneous indicator condition. That exposure duration was then used throughout the experiment for all conditions for that $S$. The mean exposure duration was $38 \mathrm{msec}$, with a range of 30 to 50 .

\section{Risults and Discussion}

In Table 1 the mean percent correct identifications of the indicated letter are shown for each of the five experimental conditions. In the three conditions where $S$ reported two letters, $R_{1}$ and $R_{2}$ in the table designate these responses in the order that $\mathbf{S}$ was instructed to make them. The overall significance of mean differences in Table 1 was evaluated by a two-way classification analysis of variance (Ss by Condition), in which the second response in each of the double response conditions was treated as a separate condition. Thus, there were eight conditions in the analysis of variance. The analysis showed a significant variation between conditions $[\mathrm{F}(7,35)=15.3, \mathrm{p}<.001]$ as well as a significant $S$ effect $[F(5,35)=12.4, p<.001]$

The effect of having to process two indicators and two letters from the display can be seen by comparing the two simultaneous indicators/two responses condition with the single simultaneous indicator/single response condition. In the former condition, the Ss were instructed to give their responses in a clockwise order. Although the S's first response under this condition is significantly more accurate than the second response, it is still significantly poorer than performance for the single response condition. 1

The decrement in performance under the two indicators/two responses condition could be due to the added load of processing two indicators, of encoding two letters, or a combination of these. However, comparison of the data of this condition with the single indicator/two responses condition suggests that the decrement is mainly attributable to encoding two letters from the display. In this latter condition, only a single indicator is involved, and $\mathbf{S}$ reports the two letters right at this average level, $79 \%$.)

The above pattern of results can be explained reasonably well in the following manner. Let us assume that encoding from the display or the transfer from the icon to a short-term memory is carried out serially, letter by letter, and that encoding itself requires a finite period of time. During the time encoding of a letter is taking place the display icon is deteriorating so that by the time the second letter is encoded the legibility of the icon is reduced. These assumptions would lead to the prediction that the second response would be less accurate than the first, which is contradicted by the data obtained in the single indicator/two responses condition. However, it is to be noted that the order in which $\mathbf{S}$ reports the letters is not necessarily the same order that he encoded them.

If the three two-response conditions are compared, it seems reasonable that the encoding order would have followed the report order most frequently in the early and simultaneous indicators condition. Here the $\mathbf{S}$ has 250 msec to process the indicator and to locate position before the display and the second indicator appear. It seems most likely that the $S$ would encode the letter in the position he had already located before turning to the second indicated letter. Only when the second indicated letter was in the immediately adjacent position of the display would there seem to be a significant probability that this letter would be encoded first.

The most frequent deviation of encoding order from report order might be expected to occur in the single indicator/two responses condition, where the indicator designates a position between two adjacent letters. Although instructed to report the letters in a clockwise order, the close adjacency of the letters might well lead to variation in the order in which they are encoded, particularly for bottom and left half display positions.

In the two simultaneous indicators condition with clockwise order of report, order of encoding would probably follow response order except for deviation again when the two designated letters occupy immediately adjacent positions or when, through random variation in the clarity of the letters in the display, the letter to be reported second stands out more in clarity (Eriksen, 1966).

This account fits the difference observed between first and second responses in the three conditions quite well. The biggest differences in accuracy between first and second responses occurred in the early and simultaneous indicators and two simultaneous indicators conditions. 
Table 2

Mean Percent Correct Letter Identifications by Response and Condition as a Function of Whether the Two Indicated Letters Were in Adjacent or Separated Display Positions

\begin{tabular}{|c|c|c|c|c|}
\hline \multirow[b]{3}{*}{ Position } & \multicolumn{4}{|c|}{ Conditions } \\
\hline & \multicolumn{2}{|c|}{$\begin{array}{c}\text { Early and } \\
\text { Simultaneous } \\
\text { Indicators, } \\
\text { Two Responses }\end{array}$} & \multicolumn{2}{|c|}{$\begin{array}{c}\text { Two } \\
\text { Indicators, } \\
\text { Two } \\
\text { Responses }\end{array}$} \\
\hline & R1 & R2 & R1 & R2 \\
\hline Adjacent & 80 & 78 & 80 & 83 \\
\hline Separated & 90 & 69 & 81 & 73 \\
\hline
\end{tabular}

The least difference occurred for the single indicator/two responses condition.

However, a further check can be made upon the assumption concerning the deviation of encoding order from report order when the letters to be reported are in immediately adjacent positions. The data from the two indicators/two responses and the early and simultaneous indicators/two responses conditions were analyzed to determine whether the accuracy of the first and second responses varied as a function of whether the two indicators designated immediately adjacent letters in the display as opposed to letters removed by one position or more.

The data in Table 2 show the result of this analysis. Percent correct identifications are shown for each of the two conditions and for each of the two responses as a function of whether the two indicators designated adjacent positions on the circular display or positions separated by at least one intervening position. A four-way analysis of variance (conditions, first and second responses, positions, and Ss) was performed on these data. The main interest was the significant interaction between first and second responses and position (adjacent or separated) $[F(1,5)=29.36, p<.01]$.

If the letter to be given in the first response has a greater likelihood of having been encoded second when the other letter to be reported is in an immediately adjacent position, it would be expected that the accuracy for the first response would increase with the separation between the stimuli and the reverse would occur for the second reported letter. This effect is confirmed quite clearly in the data for the early and simultaneous indicators condition. If the two letters to be reported occur in adjacent display positions, there is little difference in the accuracy of report as a function of the order in which $S$ reports the letters. However, if the letters are separated by at least one display position, accuracy of the first reported letter increases very markedly, whereas that of the second reported letter shows a marked decrease. A somewhat similar effect is obtained in the data for the two simultaneous indicators condition. Accuracy for the second reported letter drops rather pronouncedly when the letters are separated by at least one intervening position, although the gain in accuracy with separation for the first reported letter is not as pronounced as in the other condition.

The above explanation of the results can be extended to account for the data obtained in the opposite/single response condition. Here the $\mathbf{S}$ was instructed not to report the letter indicated but the one diametrically opposite on the circular display. Although this condition involved S's giving only one response, accuracy was significantly poorer than that obtained in the single indicator/single response condition. However, the Ss ran the experimental sessions in which this condition was intermixed with blocks of trials under the other conditions. If we assume that, due to the preponderance of their experience, they developed the habit of encoding first, on a large proportion of trials, the letter immediately designated by the indicator and then of encoding the diametrically opposite letter they were to report, the performance of the opposite/single indicator condition is explicable. Although required to report only a single letter, Ss were actually encoding two letters on a significant proportion of the trials. This would account for why performance under this condition for a single-letter response was at the level of the two-response conditions when the average performance on both responses for these conditions was considered.

\section{EXPERIMENT 2}

Since an evaluation of the data in the above experiment involved multiple comparisons within the same sample of Ss, there are problems associated with determining just how reliable obtained differences are. Accordingly, it was desirable to replicate the conditions of Experiment 1 on a new sample of Ss.

Two new control conditions were added in this experiment. The early indicator/single response condition consisted of an indicator's appearing $250 \mathrm{msec}$ before display onset. This condition was included to assist in interpreting the data from the early and simultaneous indicators/two responses condition and to insure that the gain in performance obtained with an early indicator in previous experiments was operating in the present experimental arrangements (Eriksen \& Collins, 1969). The other control condition was a delayed indicator/single response condition in which an indicator appeared $700 \mathrm{msec}$ following the termination of the display and $S$ was required to report the letter that had been in the position designated by the indicator. While the advantages of an indicator presented during the exposure of the display relative to one presented after the icon has deteriorated would seem to have been well established by now (Averbach \& Coriell, 1961, and Eriksen \& Collins, 1969), we nonetheless deemed it desirable to make certain that the effect was operating in our present experimental arrangement.

In addition to these control conditions, a modification was made in the opposite condition of Experiment 1 . In the previous experiment $\mathbf{S}$ was required to report only a single letter, the one occupying the position diametrically opposite the letter designated by an indicator occurring simultaneously with the display. In the present experiment, $S$ was instructed to report the letter immediately designated by the indicator as well as the one occupying the diametrically opposite position. Thus, this condition became a two-response condition. The rationale for this modification will be made clear in the results and discussion section.

\section{Subjects}

One male and four female undergraduate students at the University of Illinois served as paid Ss. All had normal or corrected to normal vision.

\section{Apparatus and Stimuli}

The apparatus and stimuli were the same as those employed in Experiment 1, with one slight modification. A capital $V$ was substituted for the capital $Y$ in the four stimulus letters. There had been a suggestion in the previous data that the $\mathrm{Y}$ was different in discriminability from the other three letters.

\section{Procedure}

The procedure was identical in terms of practice and experimental sessions to that of Experiment 1 with the exception that only eight experimental sessions were involved. During these experimental sessions, seven blocks of 12 trials each (one block of each condition per session) were run in counterbalanced order. Each session was preceded by $5 \mathrm{~min}$ of dark adaptation and warm-up with a single simultaneous indicator. Feedback was given on each trial during warm-up. During experimental sessions, feedback was given by telling 
Table 3

Mean Percent Correct Identifications of Indicated Letters for the Seven Conditions of Experiment 2. Also shown are the values averaged across the common conditions of Experiments 1 and 2 .

\begin{tabular}{|c|c|c|c|c|c|c|c|c|c|c|}
\hline \multirow{2}{*}{$\begin{array}{c}\text { Single } \\
\text { Indicator, } \\
\text { Single } \\
\text { Response }\end{array}$} & \multicolumn{2}{|c|}{$\begin{array}{c}\text { Single } \\
\text { Indicator, } \\
\text { Two Responses }\end{array}$} & \multicolumn{2}{|c|}{$\begin{array}{c}\text { Two } \\
\text { Indicators, } \\
\text { Two Responses }\end{array}$} & \multicolumn{2}{|c|}{$\begin{array}{l}\text { Eariy and Simul- } \\
\text { taneous Indicators, } \\
\text { Two Responses }\end{array}$} & \multicolumn{2}{|c|}{$\begin{array}{c}\text { Single Indicator, } \\
\text { Opposite, } \\
\text { Two Responses }\end{array}$} & \multirow{2}{*}{$\begin{array}{c}\text { Early } \\
\text { Indicator, } \\
\text { Single } \\
\text { Response }\end{array}$} & \multirow{2}{*}{$\begin{array}{c}\text { Delayed } \\
\text { Indicator, } \\
\text { Single } \\
\text { Response }\end{array}$} \\
\hline & R1 & R2 & R1 & R 2 & R1 & R2 & R1 & R2 & & \\
\hline 87 & 85 & 83 & 83 & 79 & 89 & 77 & 89 & 73 & 95 & 62 \\
\hline 87 & 83 & 83 & Values A & $\mathrm{Ac}$ & $\begin{array}{c}\text { Conditi } \\
\mathbf{8 9}\end{array}$ & of Expe & nts 1 & 2 & & \\
\hline
\end{tabular}

$S$ the total number of correct for each block of 12 trials. For each of the seven experimental conditions, each letter and each position were designated by the indicators equally often.

\section{Results}

In Table 3 the percent correct letter identifications are shown under each of the seven experimental conditions. For the four two-response conditions, $R_{1}$ and $R_{2}$ in the table designate first and second responses in the order that Ss were instructed to give them. The table also contains, by condition, the data averaged through Experiments 1 and 2. An analysis of variance of the Experiment 2 data, in which the second response data for the four two-response conditions were treated as four additional conditions, gave an $F$ value for between conditions of 17.0 , which, with $\mathrm{df}=10 / 40$, is significant beyond the .01 level.

If the four conditions that are the same across Experiments 1 and 2 are compared, it is seen that a satisfactory replication has been achieved. In both experiments, the simultaneous indicator/single response condition yielded $87 \%$ correct (which by itself is not too remarkable since an attempt was made to adjust the exposure duration for individual Ss to yield performance under this base condition between $85 \%$ and $90 \%$ correct). For the three two-response conditions, performance is also comparable. For the two indicators/two responses condition, there is lower accuracy for the second response as compared to the first, and both responses are poorer than those obtained for the single indicator/single response condition. The early and simultaneous indicators/two responses condition again shows slightly better performance for the first response and an appreciable deficit in second response accuracy as compared with the single indicator/single response condition. This is the same result as was obtained in Experiment 1. High correspondence across experiments is also obtained in the single indicator/two responses condition where again both responses differ little from each other in accuracy and both are lower in accuracy than that obtained with the base condition, although in this experiment the difference between these conditions is not quite as great as it was in Experiment 1.

The opposite/two responses condition was new with Experiment 2. It was employed in order to test the explanation for the data obtained in the opposite/single response condition in Experiment 1. In Experiment 1 this condition, although involving only a single indicator and a single response, had given significantly poorer performance than the single indicator/single response condition. The interpretation advanced for this result had been that the Ss, although required to report only the letter in the position diametrically opposite to the position where the indicator occurred, were nonetheless, on a large proportion of the trials, also encoding the letter immediately designated by the indicator before encoding the one opposite. The decrease in accuracy for the reported opposite letter was thus attributed to decay in the icon that occurred during the time the other letter was being encoded.

In the present experiment, the requirement that the $S$ report both letters checked upon this interpretation in the following way. If the $S$ by instruction is actually encoding the letter closest to the indicator first on all or nearly all trials, we would expect little or no deterioration of performance on this letter relative to the base condition. The spatial separation of the two encoded letters would almost always assure that this letter would be encoded first. There should, however, be an appreciable drop in the accuracy of report of the letter in the opposite position inasmuch as $\mathbf{S}$ would encode this letter second. As can be seen from the data in Table 2, this is the result. Under conditions where we can be reasonably confident in assuming that the order of report is also the order of encoding, there appears to be no decrement for the letter reported first as compared with the base condition.

If the four two-response conditions are compared on the average accuracy with which indicated letters are reported, all four conditions are found to be very comparable, with average accuracy varying from $81 \%$ to $84 \%$. This is so despite the differences in the way the two letters to be reported are indicated in these four conditions. This result is consistent with the "tradeoff" interpretation advanced above, namely, that Ss are capable of processing two letters from a display at a given level of accuracy, and variation in accuracy between letters reported first and second can be attributed to the order in which they were encoded into short-term memory. The more consistently Ss use the prescribed order for encoding, the greater difference there will be between the two letters. The comparable average accuracy across conditions also suggests that the method of attempting to program or direct selective memory within the operations of these four experimental conditions is relatively ineffective in the sense that it failed to improve the S's information handling capacity. This, of course, implies that having two separated indicators each designating a separate element, having a single indicator designating two adjacent display elements, or even a single indicator designating two elements separated by the maximum distance in the display can be equally effective in directing selective attention.

Since the performance difference for the two responses in the simultaneous and opposite/two responses condition can be accounted for adequately in terms of the order in which the stimuli are encoded, it would appear that there is no basis for assuming that time is required for the selective encoding process to move spatially from one display pasition to another. The relatively lower accuracy of the second responses of this condition is most likely not attributable to the time required for a central processing or encoding mechanism to go diametrically across the display, but is most readily explained in terms of the increased probability that the opposite item will be encoded second on nearly all trials.

In this connection, there is a slight tendency for the single indicator that 
occurs between two letters to show superiority consistently over the other two response conditions in terms of the average accuracy of the two responses. While the consistent superiority of this condition is not significant, it is suggestive-it might be attributable to decreased location errors due to the two letters to be reported being immediately adjacent to one another.

The result obtained with the delayed indicator/single response condition confirms that the experimental arrangements that were employed in these two experiments were sufficient to produce an overload of information and that a simultaneous indicator did indeed show selective effects on encoding from iconic memory. When the indicator occurs 700 msec after termination of the display, it can be presumed that the icon has deteriorated beyond any usable level. Thus the $62 \%$ accuracy obtained under this condition represents the probability of having tapped one of the few items that $S$ was able to encode from the display during its presentation and the duration of the usable icon.

The early single indicator/single response condition is found to lead to an appreciable increment in performance over that obtained for a single simultaneous indicator. This is consistent with previous findings (Erikgen \& Collins, 1969). Its importance for the present experiments, however, is in terms of interpretation of the early and simultaneous indicators/two responses condition. In both Experiments 1 and 2 , the first responses obtained under this experimental condition were at the same level of accuracy as that for the single indicator/single response condition. In interpreting this effect as attributable to the S's sometimes encoding the second indicated response first, particularly on trials where it was adjacent to the first indicated response, we were assuming that there should have been a gain for the first or early indicated response relative to the single indicator/single response condition. If $\mathbf{S}$ had time to locate the display position or to spatially structure the display for encoding prior to the onset of the stimulus letters, performance for the prior designated letter would be better than that obtained with the single simultaneous indicator. The analysis contained in Table 2 supported this interpretation, but it seemed desirable to determine for sure that the gain from an early indicator that was assumed to underlie this explanation was indeed obtained in the present experimental arrangements. The findings for the early indicator condition in Experiment 2 are confirmatory.

Both Collins (1970) and Gardiner (1970) have found that average response accuracy decreases when $\mathrm{Ss}$ are required to report two or more letters from visual displays. As Gardiner points out, this reduction in accuracy per se cannot unequivocally be attributed to a serial processing or encoding of the display information since limited capacity, parallel processing models such as Rumelhart's (1970) predict such an outcome. The reduction in performance with two or more items can be attributed to the distribution of the limited capacity over all the items which are simultaneously, but less adequately. processed.

The present results provide much less equivocal evidence for serial encoding. To the extent that our inferences are sound as to the Ss' encoding order of the display letters, a serial model is indicated. The first item, or letter, encoded on a multiple item encoding task is as efficiently and accurately processed as when the task requires encoding only a single item. This is contrary to what would be expected from a limited capacity parallel model such as Rumelhart's when extended to encompass the Collins (1970) and Gardiner (1970) results.

While a serial encoding of the display letters is supported, the possibility remains that indicator processing and position location in the display can occur simultaneously with letter encoding. In view of the evidence that apprehension of the indicator and location of display position requires between 200 and $300 \mathrm{msec}$ (Eriksen \& Collins, 1969; Averbach \& Coriell, 1961) performance much worse than that obtained would have been expected for the second response in the two indicators/two responses condition. If 200 msec was required to locate the second letter after the first letter had been selected and encoded, appreciable deterioration in the icon would have occurred. Yet, average performance across the two responses in this condition was not appreciably lower than that obtained for two responses when only one indicator and location were presented (single indicator/two responses condition). An alternative to simultaneous processing of indicator and position while encoding letters is to consider the possibility that the 200 or more milliseconds required to apprehend an indicator and determine position reflects a spatial structuring process. Once this structuring is accomplished, encoding can move about the structured field with no further time required for position location. The present results do not rule out a possibility of this sort.

\section{REFERENCES}

AVERBACH, E., \& CORIELL, A. S. Short-term memory in vision. Bell system Technical Joumal, 1961, 40. 309-328.

COLLINS, J. F, Correlations in sensitivity on different foveal areas. Unpublished doctoral dissextation, University of Ilinois, 1970.

ERIKSEN, C. W. Independence of successive inputs and uncorrelated error in visual form perception. Journal of Experimental Psycholosy, 1966, 72, 26-35.

ERIKSEN, c. W. \& COLLINS, J. F. Temporal course of selective attention. Journal of Experimental Psychology, $1969,80,254-261$.

ERIKSEN, C. W., \& ROHRBAUGH, J. W. Some factors determining efficiency of selective attention. American Joumal of Psychology, 1970, 83, 330-342.

FLOM, M. C., WEYMOUTH, F. W., KAHNEMAN, D. Visual sesolution and contour interaction. Journal of the Optical Society of America, 196, 53, 1026-1032.

GARDINER, G. T. Spatial processing characteristics in the perception of brief visual arrays. Unpublished doctoral dissertation, University of Michigan. 1970.

KEELEY, S. M. Visual detection as a function of attentional demand and perceptual system error. Unpublished doctoral dissertation, University of Iinois, 1968.

RUMELHART, D. E. A multicomponent theory of the perception of briefly exposed visual displays. Joumal of Mathematical Psychology, 1970, 7, 191-218.

SHAW, P. Processing of tachistoscopic displays with controlled order of characters and spaces. Perception \& Psychophysics, 1969, 6, 267-266.

SPERLING, $G$. The information available in brief visual presentations. Psychological Monographs, 1960, 74(11, Whole No. 498).

\section{NOTE}

1. Evaluation of the statistical significance of difference between percentages is complicated by the fact that multiple comparisons are being made on the same cample of Ss. As a rough rule a difference will be considered "significant" if it equals or exceeds the mean difference (5\%) for the .01 level on a two-tailed test. The alarmed statistical conservatives may be reasaured by turning to Experiment 2, where the important differences have been replicated on an independent sample.

(Received for publication December 12 , 1970.) 